\title{
Towards Context-aware Process Guidance in Cyber-Physical Systems with Augmented Reality
}

\author{
Klaus Kammerer ${ }^{1}$, Rüdiger Pryss ${ }^{1}$, Kevin Sommer ${ }^{2}$, Manfred Reichert ${ }^{1}$ \\ ${ }^{1}$ Institute of Databases and Information Systems, Ulm University, Germany \\ Email: \{klaus.kammerer, ruediger.pryss, manfred.reichert\}@uni-ulm.de \\ ${ }^{2}$ Uhlmann Pac-Systeme GmbH \& Co. KG, Laupheim, Germany \\ Email: sommer.k@uhlmann.de
}

\begin{abstract}
Assembly, configuration, maintenance, and repair processes in cyber-physical systems (e.g., a press line in a plant) comprise a multitude of complex tasks, whose execution needs to be controlled, coordinated and monitored. Amongst others, a process-centric guidance of users (e.g. service operators) is required, taking the high variability in the assembly of cyberphysical systems (e.g. press line variability) into account. Moreover, the tasks to be performed along these processes may be related to physical components, sensors and actuators, which need to be properly recognized, integrated and operated. In order to digitize cyber-physical processes as well as to guide users in a process-centric way, therefore, we suggest integrating process management technology, sensor/actuator interfaces, and augmented reality techniques. The paper discusses fundamental requirements for such an integration and presents an approach for process-centric user guidance that combines context and process management with augmented reality enhanced tasks. For evaluation purposes, we analyzed the cyber-physical processes of pharmaceutical packaging machines and implemented selected ones based on the approach. Overall, we are able to demonstrate the usefulness of context-aware process management for the flexible support of cyber-physical processes in the Industrial Internet of Things.
\end{abstract}

Index Terms-Cyber-physical System, Context-aware Process Management, Augmented Reality Enhanced Process, Cyberphysical Process

\section{INTRODUCTION}

The Industrial Internet of Things (IIoT) has become increasingly pervasive in industry as digitization is a business priority for almost every enterprise. In particular, the IIoT is essential for realizing the Industry 4.0 vision, which shall enable new opportunities in the digital transformation of manufacturing, integrating smart machines, advanced analytics, and people at work [1]. In this context, the building blocks of the IIoT, the so-called cyber-physical systems (CPS), shall allow bridging the physical and digital world and, thus, enable innovative digital services in manufacturing as well as laying the foundation for smart plants and digital factories.

For the assembly, configuration, maintenance, and repair of CPS, complex procedures-denoted as cyber-physical processes in the following-exist. The latter comprise both human and technical tasks, whose execution needs to be controlled, coordinated and monitored. In this context, a process-centric interaction between users and CPS is essential for bridging the gap between digital and physical process. Moreover, the complex tasks of a cyber-physical process may be related to the physical components, sensors and actuators of a CPS, which therefore must be properly recognized, integrated and operated. While some of the tasks need to be performed manually, others can be fully automated (e.g., collecting sensor data on the machine) or be enhanced with digital services (e.g., maintenance enhanced with augmented reality).

Although the potential of digitizing cyber-physical processes has been recognized in literature [2], the role of business process management (BPM) technology in the digital transformation of manufacturing has not been well understood so far. In general, cyber-physical processes are knowledge-intensive and their proper support plays a key role in bridging the gap between physical systems and their digital representations.

As a particular challenge, the variability of cyber-physical processes needs to be properly handled [3]. The latter is caused by the variety of configurations that may exist for the assembly of a CPS. Think of a press line, for example, whose concrete stations, hardware components, and software modules may depend on contextual factors like customerspecific requirements, legal regulations, or other site-specific factors. As a consequence, there exists a high variability of the processes for the assembly, configuration, maintenance or repair of CPS, i.e., the concrete tasks of a cyber-physical process and the digital data (e.g., checklists, instructions, or videos) provided during task execution may vary depending on contextual factors. Note that this process variability introduces a huge complexity for service workers, particularly when facing CPS that run over years or decades. Finally, in certain cases, cyber-physical processes may have to be dynamically adapted during runtime, e.g., to react to contextual changes (e.g., retooling of a machine) or to cope with faulty sensors and actuators.

Another fundamental challenge in bridging the gap between physical and digital CPS components is to enhance the sense of workers for their physical environment with the help of digital data [4]. In this context, the integration of cyberphysical processes with augmented reality (AR) technologies offers promising perspectives, i.e., user guidance for assembly, configuration, maintenance, and repair processes of CPS will benefit from an AR enhanced task support [5]. In consequence, the process-centric guidance of service operators based on the enhancement of physical components with digital data should be a key feature of any CPS [6]. Note that the instructions 
for performing the technical tasks of a cyber-physical process are more comprehensible if they are not only available as paper manual, but also as digital 3D drawings superimposed upon the actual physical system, showing step-by-step the tasks to be performed and the way how to perform them [7], [8]. Moreover, sensor data and task guidelines might be incorporated through the AR interface of the service operator in real time, and data be overlaid on and registered with the actual equipment in his field of view.

In previous work, we developed advanced methods, concepts and technologies that enable full life cycle support of digital business processes [9], [10], [11], [12]. In principle, respective techniques can be also applied to cyber-physical processes, but need to be enhanced in order to meet the specific requirements of the latter. Besides sophisticated configuration facilities and AR-enhanced process guidance, advanced techniques for the proper integration of human resources, sensors and actuators with the digital processes are needed. This paper gives first insights into the support of cyber-physical processes and the particular challenges imposed by them. In detail, the contributions of the paper are as follows:

- We present a CPS maintenance process from the field of pharmaceutical packaging, which we discovered in a case study, and discuss its characteristics.

- Taking this real-world cyber-physical process scenario, together with insights from a literature survey, we derive fundamental requirements for the design, implementation and automation of cyber-physical processes.

- We present core components (i.e., context-aware process injection, context modeling, and AR-enhanced process guidance) of an approach that allows for the flexible specification, configuration and enactment of cyber-physical processes, taking contextual factors (e.g., the CPS variant the process refers to or individual preferences of service operators) into account as well.

We consider these contributions as an important step for the evolution of process management technology and its broader use in the context of CPS.

Underpinning our research, we applied the design science research methodology [13]. In particular, our work can be categorized as a design- and development-centered approach. By analyzing a real-world application scenario (cf. Section II) and by evaluating existing approaches (cf. Section III), we derived requirements and solution objectives (cf. Section IV), and then iteratively elaborated concepts to address the requirements (cf. Section V). Section VI concludes the paper with a summary and outlook.

\section{Cyber-Physical Processes}

To reveal basic challenges of cyber-physical processes, we conducted a case study in the field of mechanical engineering. During this case study, we identified, discovered and analyzed scenarios dealing with maintenance processes of a press line for pharmaceutical packaging, i.e., a CPS used to pack drugs (e.g., tablets) into blister packs, blister packs into boxes, and boxes into cartons. The CPS consists of interconnected machines like a forming station (cf. Fig. 1a) to form blisters into an aluminum or plastic foil or a filling station (cf. Fig. 1b) to add tablets to the blister packs. Each station, in turn, comprises various devices like a forward feeder or a deflection pulley (cf. Fig. 1c) to lead the foil through the machine. Moreover, all machines are equipped with sensors and actuators that are controlled by a programmable logic controller (PLC). Finally, all machines may be configured and controlled with the help of a touch-sensitive human machine interface (cf. Fig. 1d).

\section{Case Study: Maintenance Processes of a Press Line}

We analyzed the maintenance process of the Uhlmann B1440 Blister Packaging Press Line, which has to be periodically executed by a service operator (cf. Fig. 2). The process is triggered if a certain time period has passed since the previous maintenance cycle, certain hardware components have been used for a particular number of machine cycles, or any error occurs.

Initially, the service operator receives the maintenance plan of the press line as well as information on the maintenance intervals of its various parts (cf. Fig. 2a). Based on this information, the operator defines a task list, which may comprise hundreds of tasks to be processed during maintenance. For every task, in turn, a specific checklist or handbook guiding the operator exists. Furthermore, the service operator has to check basic constraints that need to be obeyed to ensure a correct execution of the maintenance process. Finally, he informs the operating staff about the upcoming maintenance and appoints a maintenance supervisor. Just before starting with maintenance, the operator checks his technical equipment, puts the press line out of operation, removes production goods, and prepares the operating system of the stations. Furthermore, he cleans the press line, e.g., by removing dirt and lubricants from screw connections, which otherwise might contaminate other parts during maintenance. Following these preparatory steps, the operator starts with the processing of the task list. Note that the latter may vary depending on contextual factors, e.g., the type of the press line or customer-specific service agreements (cf. Fig. 2b).

As an example of a maintenance task consider the calibration of a forming station, which is needed to adjust material spacing between heating station and cover plate (cf. Fig. 2c). When processing this task, a number of (manual) sub-tasks (e.g., removing covers and guide plates) have to be performed. In addition, the heating circuit has to be cooled down to avoid skin burns (cf. Fig. 2d). Finally, during the maintenance process, the machine is partially running in a test production mode with reduced speed (cf. Fig. 2e).

Note that the various maintenance tasks may refer to 


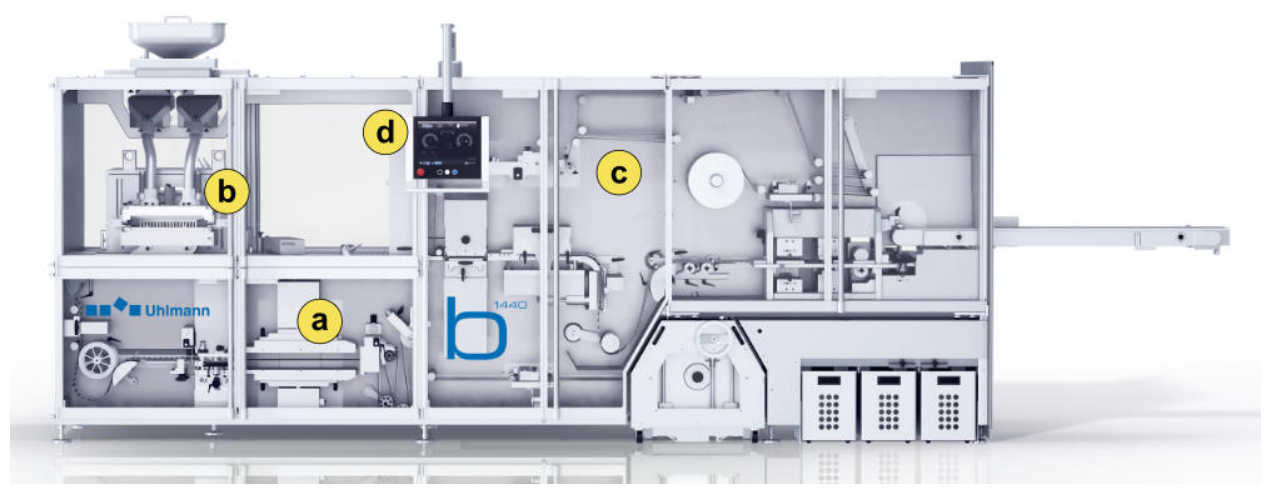

Fig. 1. Uhlmann Blister Press Line B1440

different stations of the press line. For most parts, the tasks related to different stations may be executed independently from each other. However, in certain cases, there exist subtle task interdependencies that need to be considered for proper process completion. For example, the replacement of a roll mounting requires its cleaning after test run execution (cf. Fig. 2f).

If the machine operator reports specific issues, the respective station has to be examined in greater detail. Every change applied to the station in this context has to be recorded. After completing maintenance, the press line has to be cleansed again according to a pre-specified procedure and lubricants have to be supplied to moving parts of the machines. Finally, the service operator documents all executed tasks.

\section{RELATED WORK}

The first category of related work deals with the use of AR technologies in the context CPS maintenance. In many industries, the efforts for maintaining complex machines and systems could be reduced when using AR techniques. For example, [8] were able to show that the replacement of paperbased assembly instructions with AR enhanced ones led to a significant reduction of assembly times. In turn, HUDSET [6] supported factory workers with a head-mounted display during the process of manufacturing an aircraft; on one hand, AR support was highly welcome by workers, on the other its introduction raised many technical issues. KARMA, an AR application for laser printer maintenance [7], was considered as useful with respect to maintenance processes. In turn, ARMAR [5] investigated the effects of applying realtime overlays to the machine components to be maintained. Amongst others, for the field of vehicle maintenance it was shown that ARMAR allows for an intuitive and satisfying experience of service operators. Considerable research on the use of AR technologies in industrial environments was conducted by the ARVIKA consortium [14]. A particular feature developed in the context of this initiative, was the workflow-driven selection and presentation of information to service operators. As opposed to our approach, however, only hard-coded and static workflows (i.e. executable processes) were considered. Altogether, all mentioned AR approaches either lack process management support for manual tasks or show severe limitations regarding the context-based support of process variants.

The second category of related work we consider stems from the BPM field and deals with the challenges that arise in the context of the Internet-of-Things (IoT). In particular, [2] presented a catalogue of challenges that need to be tackled when combining business process management with the IoT. For example, in cyber-physical systems, various types of tasks require the interplay between human operators and software modules, which should be coordinated by a BPM system. Therefore, an appropriate mapping from process activities (i.e. tasks) to visualizations is needed allowing operators to perform their work in an intuitive way.

Other related work deals with the context-driven execution and adaption of business processes. For example, [15] presents an automated approach for the context-aware extension of running process instances in order to flexibly cope with process variability and to increase process flexibility. Related approaches deal with the configuration of process models before deploying them to any execution platform (i.e. process engine) [16], [17], the adaptation of running process instances in the midst of their execution [9], the late selection of subprocesses during the execution of a superordinate process [18], and the late composition of process-centric services [19]. However, these approaches do not consider any contextaware AR enhanced process guidance as in our approach. Moreover, they neither use contextual models nor contextual data sources (e.g., CPS sensors) for configuring and adapting running processes.

To enable contextual awareness in business process management, process models and contextual models need be tightly integrated. [20] presented an approach for the modeling of business processes that support context descriptions. A taxonomy was introduced, which comprises contextual elements related to location, time, human resources, and organizational entities in order to integrate context related knowledge into business processes. Additionally, a concept for context-aware 


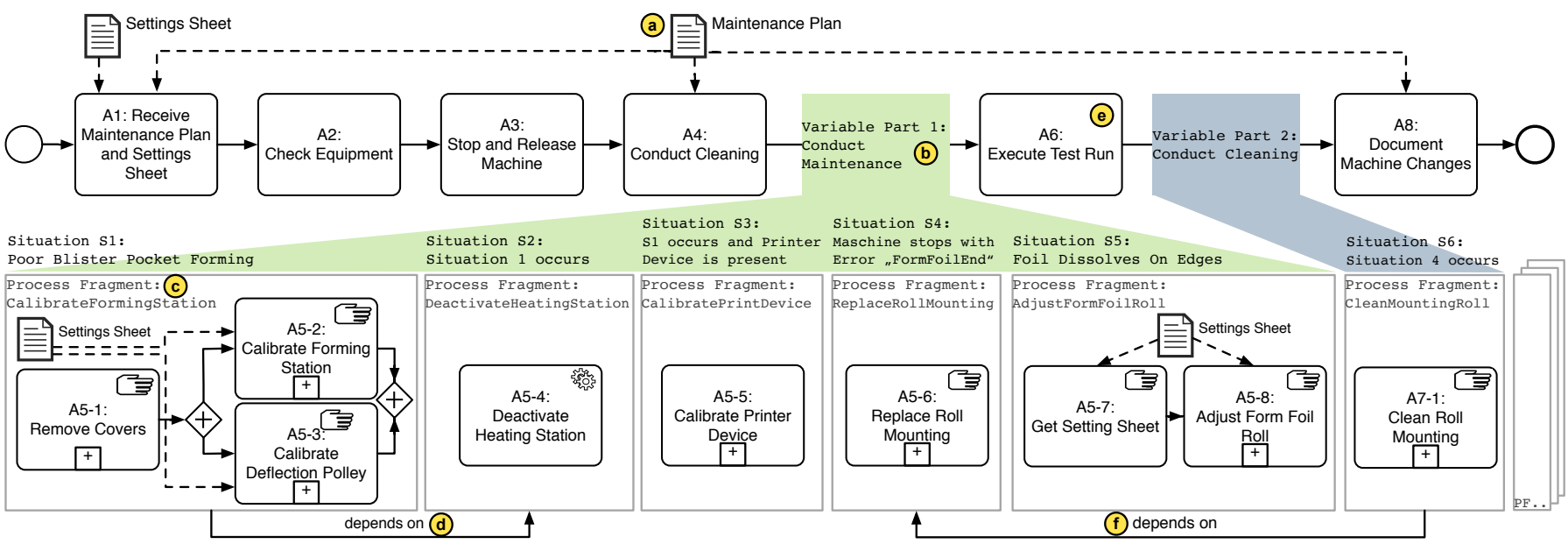

Fig. 2. Excerpt of a Machine Maintenance Process Model (Simplified Views)

process instance creation was introduced. However, contextrelated knowledge is evaluated prior to process instance creation rather than during process instance execution. The latter, however, is of utmost importance to be able to adapt a running process to evolving or changing context-related knowledge. Finally, [21] proposed a framework considering various context types and their integration with business processes, e.g., including a goal-oriented process modeling approach for context classification, formalization, and integration.

When characterizing contextual data, constraints of a certain data set are often described based on contextual situations. According to [22], contextual situations can be defined as a semantic abstraction of low-level data . Furthermore, [22] defined constraints that need to be evaluated against lowlevel data to indicate the execution of certain actions enabling situational awareness. In turn, [23] presented a rule-based situation management framework to facilitate the design of situation-aware applications and to manage contextual data. However, this work rather focused on user conditions than business processes. Finally, [24] presented an approach characterizing the context of a business process activity through conceptual models structured by contextual, process-, and domain-specific ontologies. However, this approach did not describe any concepts for integrating these conceptual models with BPM systems.

\section{REQUIREMENTS}

Taking the application scenario from Section II and the insights we gained when studying the limitations of related work, we identified three categories of requirements for the support of cyber-physical processes, i.e., context-aware processes, context management, and augmented reality support (cf. Table I).

The maintenance process of a CPS may comprise hundreds of tasks, whose composition varies depending on contextual factors (see Requirements $C l-1$ and Cl-2). For example, devices may show different wear marks depending on the previous degree of utilization. Moreover, a CPS may have
TABLE I

REQUIREMENTS

\begin{tabular}{|c|c|c|}
\hline No & Title & Description \\
\hline \multicolumn{3}{|c|}{ Category 1: Context-aware Processes } \\
\hline C1-1 & Variability Support & Support for flexible modeling \\
\hline $\mathrm{C} 1-2$ & $\begin{array}{l}\text { Execution \& Adap- } \\
\text { tation }\end{array}$ & Run time adaptations \\
\hline $\mathrm{C} 1-3$ & Traceability & Logging \\
\hline \multicolumn{3}{|c|}{ Category 2: Context Management } \\
\hline $\mathrm{C} 2-1$ & Modeling & Representation of a context model \\
\hline $\mathrm{C} 2-2$ & Sensor Integration & $\begin{array}{l}\text { Integrate sensor data into the context } \\
\text { model }\end{array}$ \\
\hline $\mathrm{C} 2-3$ & Evaluation & Evaluate contextual situations \\
\hline \multicolumn{3}{|c|}{ Category 3: Augmented Reality Support } \\
\hline $\mathrm{C} 3-1$ & Interaction & Interaction between users and a CPS \\
\hline $\mathrm{C} 3-2$ & Attention & Guidance of a user's attention \\
\hline $\mathrm{C} 3-3$ & Mapping & $\begin{array}{l}\text { Mapping of physical and virtual ob- } \\
\text { jects }\end{array}$ \\
\hline
\end{tabular}

been customized to the needs of individual applications; e.g., press lines in pharmaceutical packaging and, hence, related processes vary due to different kinds of drugs to be packaged. To meet Requirements $C 1-1$ and $C 1-2$, we introduce contextaware process injection (CaPI) as fundamental concept, which allows tailoring process execution to the execution context of the respective cyber-physical process. In certain scenarios, the execution of cyber-physical processes needs to be traceable as well (see Requirement C1-3). Regarding pharmaceutical packaging, for example, traceability support for changes during packaging is mandatory due to the Title 21 Code of Federal Regulations (CFR) Part 11. The latter defines guidelines for the use of electronic records and signatures for pharmaceutical and medical devices [25]. To meet Requirement C1-3, therefore, the proposed artifact will record manually as well as (semi-)automatically executed process tasks.

In order to enable tailoring of cyber-physical processes to other contextual factors, like the execution state of a pharmaceutical machine or the current location of a technician, we introduce the concept of context graphs. The latter allows modeling data entities, which are semantically related (cf. 
Requirement $C 2-1)$. The location of a technician, for example, has to be mapped to the current process execution context. Furthermore, sensor information, such as the location of a technician and the physical structure of a machine, must be integrated into a contextual model and mapped to modeled contextual factors (cf. Requirement C2-2). Sensed data then has to be evaluated in order to decide whether a given contextual situation of the CaPI concept is present to be able to tailor process execution of the considered cyber-physical process (cf. Requirement $C 2-3$ ). Note that in current practice, task-related information for service operators is provided conventionally, e.g., procedural instructions for machine maintenance can be found in handbooks, whereas information about the current state of a machine is provided on its terminal (cf. Fig. 14). Finally, the sub-tasks to be performed in the context of a particular maintenance task are summarized in paper-based checklists.

To optimize the interaction between users and CPS (see Category $C 3$ ) and to bridge the gap between physical system and its digital representation, the proposed approach shall enhance the tasks of cyber-physical processes with AR-enabled user interfaces (cf. Requirement C3-1), i.e., the various kinds of digital information shall be presented to users in a AR view. The latter requires precise leading of a users attention (cf. Requirement C3-2). Finally, mapping of physical objects, i.e. a machine part, and its virtual counterpart in the context graph constitutes a crucial task for enabling precise interaction and attention guidance (cf. Requirement C3-3).

\section{CONCEPTS}

The support of cyber-physical processes involves several steps. Sensor data needs to be collected, processed and mapped to a context model (cf. Fig. 3a). The latter, in turn, is integrated into a context graph (cf. Fig. 3b), and a context-aware process environment (CaPI, cf. Fig. 3c). Finally, AR techniques are added to optimize the interaction between user and CPS, e.g., by enabling gesture-based controls (cf. Fig. 3d). In the following, we present concepts for context models, CaPI, and augmented reality-based interaction between users and CPS.

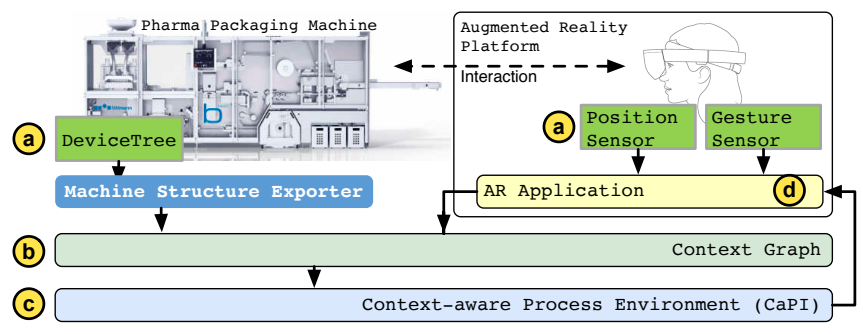

Fig. 3. Component Architecture

\section{A. Context-aware Process Injection}

To systematically support context-aware process variants as well as context-driven process adaptations at run time, we adopt the concept of context-aware process injection (CaPI) [15]. The key objective of CaPI is to ease the modeling of a collection of process variants at design time as well as to automate context-driven process adaptions at run time. Taking the current context into account, $\mathrm{CaPI}$ enables the late injection (i.e., insertion) of process fragments into a base process. The core artifact of $\mathrm{CaPI}$ is the context-aware process family $(\mathrm{CPF})$ (cf. Fig. 4). More precisely, a CPF comprises a base process model (cf. Fig. 4g) with extension areas (cf. Fig. 4h), contextual situations (cf. Fig. 4e) characterized through process parameters (cf. Fig. 4d), a set of process fragments (cf. Fig. 4i) that may be injected at specific extension areas during run time, and a set of injection specifications (cf. Fig. 4f). The latter define process fragments to be injected at certain areas when a given contextual situation applies.

Following the separation of concerns design principle, the base process model solely contains decisions and activities shared by all variants of the process. In particular, these activities need to be known at design time, and must not be changed during run time. By contrast, extension areas represent the dynamic parts of the process. Accordingly, process modelers may first model the predictable parts of the process and then add the varying parts to the base process step by step. Extension areas can then be used to automatically inject process fragments into the base process during run time, based on the respective contextual situation as well as injection specifications. Moreover, an extension area allows for the dynamic injection of any number of process fragments arranged in parallel branches. In turn, contextual situations are defined through conditions expressed in first-order logic. These conditions can take process parameters as well as data objects of the base process model into account. In this context, process parameters may be linked to external factors, i.e., context properties of a context graph (cf. Fig. $4 a+b$ ), influencing the decisions on process injections. When injecting process fragments, CaPI takes care of correct data flow mappings as well, i.e., data objects of an injected process fragment (cf. Fig. 4i) are automatically connected to existing data objects of the base process. CaPI enables dynamic configurations and changes of varying processes in a controlled way during run time. By solely enabling insertions of process fragments, it further allows process modelers to focus on the commonalities of all variants and varying process parts instead of coping with a complex process model covering all variants. Furthermore, process modelers may directly integrate contextual factors into the modeling of process variants. Thereby, external context factors are abstracted by meaningful process parameters and reusable contextual situations. CaPI is able to cope with context-driven run-time changes based on the late evaluation of contextual parameters at given extension areas. Finally, the automated construction of a consistent data flow between the injected process fragments and the base process mitigates the efforts of involved users.

\section{B. Context Modeling}

The context model for the design of the proposed artifact has been adopted from [24]. In detail, as semantic data model we generate a context graph that comprises context entities 


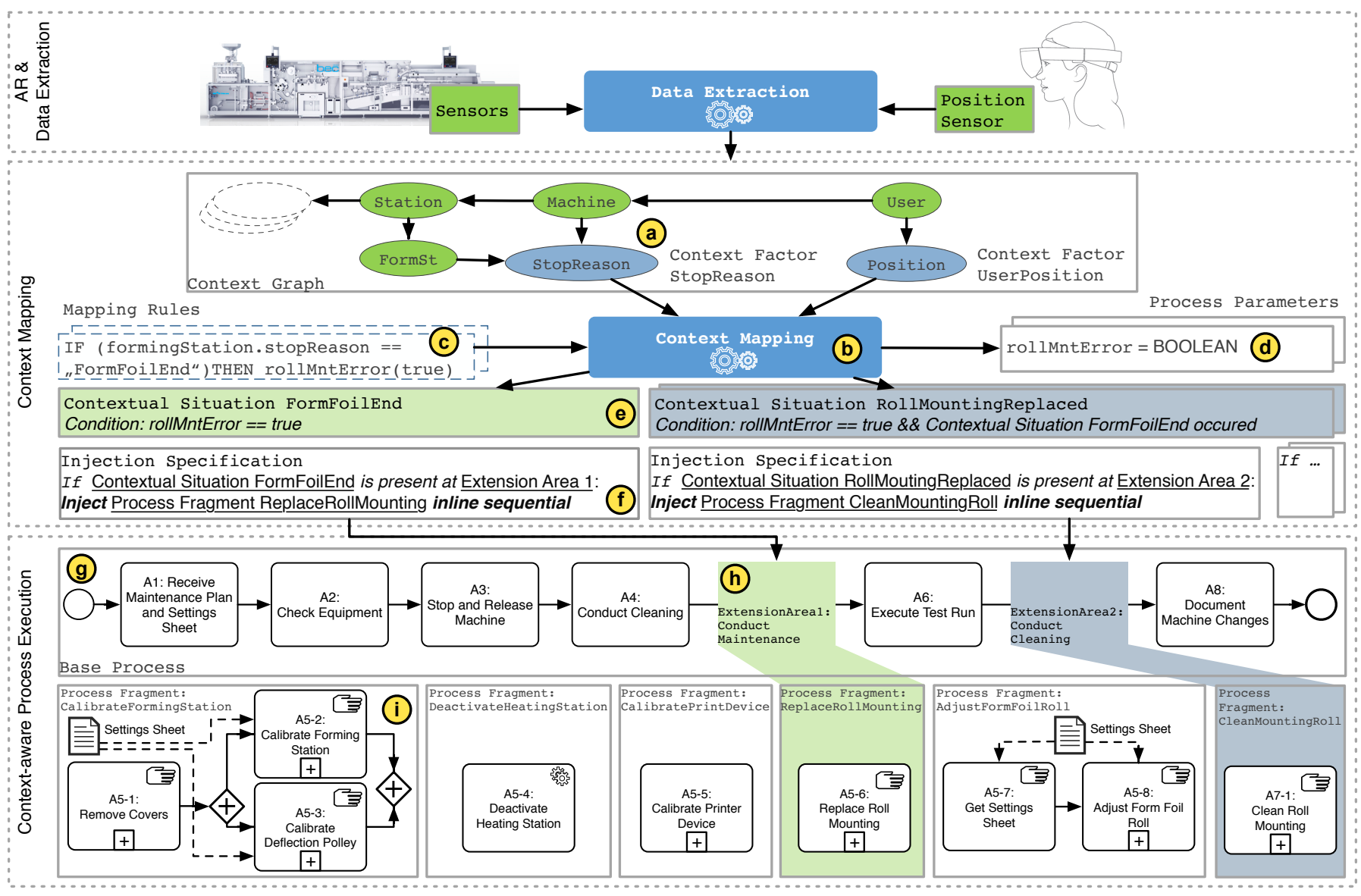

Fig. 4. Example of a Context-aware Process Family

and their properties as node types. Relations between context entities and context properties are represented as relationship edges. Every node, in turn, is associated with one of the following context types: location, time, resource, or organization, e.g., physical objects may be modeled as entities with context type resource (cf. Fig. 5a). The current state of a context entity is expressed by context properties. For example, the power consumption of a forming station in a press line is a property of a corresponding context entity (cf. Fig. 5b), i.e., the machine or the device itself depending on how precisely the context model is specified. A relation between context entities is modeled through an entity relation, i.e., an edge in the context graph that may be associated with attributes characterizing the relation (cf. Fig. 5c). Furthermore, temporally occurring relations between context properties can be modeled by virtual relations, e.g., if the current actor in the application context is the maintenance operator of a machine represented as context entity (cf. Fig. 5d).

Regarding the considered application scenario, the structure of the packaging press line is mapped onto context entities that can be automatically derived from the data model of the press line-the latter describes the CPS structure as a Device Tree Structure. Following the context model creation, the sensor data acquired with the Machine Structure Exporter (cf. Fig. 3a) is mapped onto context properties. When a context property changes, the context graph is adapted accordingly by updating the value of a context property or changing the relation of a context entity. The state of one or more elements of the context graph (i.e., context entities or properties) is described by a contextual situation (cf. Fig. 5e). The latter corresponds to a set of predicates, i.e., context mapping rules (cf. Fig. 5f). Note, that mappings between context nodes and contextual situations are illustrated as situational relations (cf. Fig. 5g).

\section{Supporting Interactions Between Users and CPS}

The sense of users for the CPS as well as for the manual tasks of cyber-physical processes can be enhanced by integrating the latter with augmented reality (AR) technology, i.e., the goal is to provide process-centric user guidance in accomplishing complex tasks related to physical components of the CPS.

In general, AR enriches the user's view of the physical environment with virtual (i.e. digital) objects overlaid and integrated into this view, and thus it virtually enhances the user's perception of the CPS. Contemporary AR usually uses live video images being digitally enriched with computer-generated graphics. Two fundamental steps have to be accomplished in this context [26]: First, the current state of the physical 


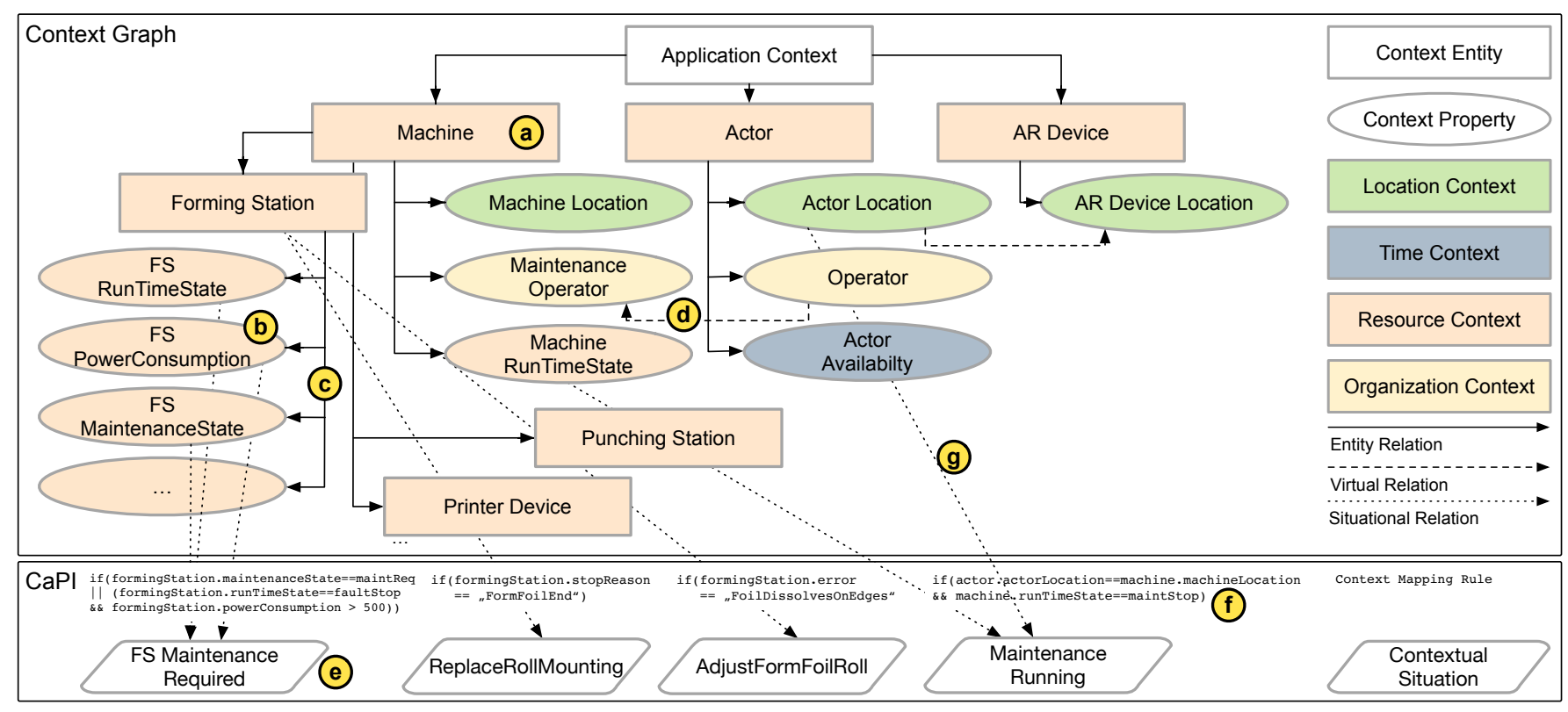

Fig. 5. Context Graph Model

world and, based on this, the state to which the virtual world (i.e., the virtual enhancements) needs to be transferred must be determined. Second, the virtual enhancements have to be displayed in a way, together with the physical environment, such that users can sense it as part of this environment. Basically, three system components are needed [26], i.e., sensors to collect information about the current state of the physical world, processors to process the sensor data and to determine the state of the virtual world, and displays to allow users to immerse into the combined physical and virtual world. The most common AR displays are visual displays [27], which can be classified into optical see-through (OST) and video see-through (VST) displays. The former allow the wearer to perceive the physical world with their eyes with the virtual enhancements being overlaid via a holographic optical element or semi-silvered mirrors, whereas the latter do not directly let provide an enhanced camera video stream to them.

To track the camera position and create an environmental model of the CPS, easily detectable fiducial markers can be used, which are placed in the physical environment. With the help of computer vision techniques, these markers can be detected based on their visual features (i.e. marker-based tracking) [28]. On the other hand, marker-less tracking, as for example used by Microsoft's HoloLens ${ }^{1}$, automatically maps the surrounding of the user (i.e. the service operator in our case) by extracting visual features and by generating an environmental 3D model. Additionally, markers can be mapped to the CPS to be able to automatically identify physical parts during the execution of the cyber-physical processes. Further, the user's current location-in relation to the CPShas to be sensed based on visual marker-based detection. The AR application reads the marker identifier and maps

\footnotetext{
${ }^{1}$ https://www.microsoft.com/en-us/hololens/hardware
}

the user's current location to the one of the CPS in the context graph. Detected parts are then integrated into the context graph as context properties and contextual situations can be triggered (e.g., to start new process instances or change currently running ones).

Finally, a graphical user interface presented on a AR OST display, in conjunction with gesture recognition, allows executing process instances by invoking respective methods of the process execution interface. In doing so, a user's attention of shown virtual objects has to be guided. For example, a animated arrow can indicate the current point of interest of a CPS (cf. Fig. 6).

\section{Summary \& OUTLOOK}

This paper presented fundamental requirements for an artifact, which shall enable a context-aware guidance of cyberphysical processes. The artifact allows creating a context graph to manage and structure sensor data of a CPS. Further, it enables the context-driven injection of process fragments into a given base process (i.e., a basic template of the cyber-physical process) during run time. Furthermore, the interaction of users with the cyber-physical processes is supported through an AR application, which integrates position and marker sensors into the context graph. In particular, detected objects can be enhanced with digital data, enabling an improved guidance during process execution. In addition, new process instances may be started depending on the actual contextual situation, e.g., when a user picks a machine part with a specific marker. Finally, the tasks to be executed may be selected based on the current context of the user. Taking the maintenance process of a press line as representative of a cyber-physical process, we discussed the challenges to be tackled. Further, we showed how the information about the physical and logical structure of a press line can be integrated into a context graph. 


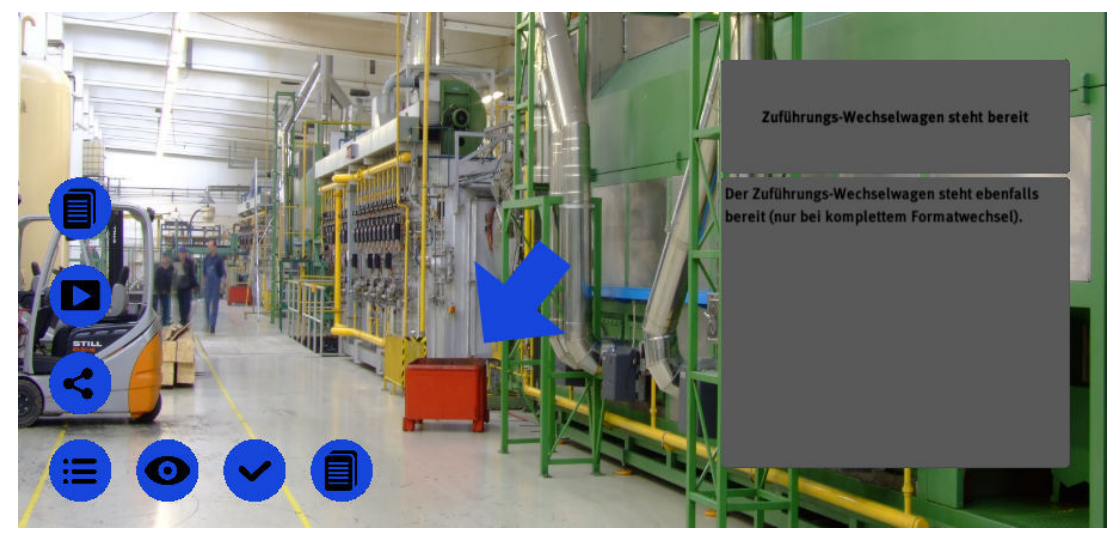

Fig. 6. Augmented Reality User Interface

In summary, linking AR with CPS offers promising perspectives for a process-centric guidance of CPS users. Sensed information about the CPS and the user, as well as the environment, allow for a tailored support of cyber-physical processes, which can be adjusted to the respective contextual situations. This way, manual tasks can be guided and monitored, enabling a much better traceability. In future work we will consider additional CPS application scenarios as well as the challenges and requirements imposed by them. Moreover, we will consider collaborative cyber-physical processes, involving multiple users and resources, as well as AR enhanced immersive analytics scenarios relevant for condition monitoring in CPS.

\section{REFERENCES}

[1] H. Lasi, P. Fettke, H.-G. Kemper, T. Feld, and M. Hoffmann, "Industry 4.0," Business \& Information Systems Engineering, vol. 6, no. 4, pp. 239-242, 2014

[2] Janiesch et al., "The Internet-of-Things Meets Business Process Management: Mutual Benefits and Challenges," Computing Research Repository, no. 709.03628, pp. 1-9, 2017.

[3] A. Hallerbach et al., "Capturing Variability in Business Process Models: The Provop Approach," J Software Maintenance and Evolution: Research and Practice, vol. 22, no. 6-7, pp. 519-546, November 2010.

[4] D. Gorecky, M. Schmitt, M. Loskyll, and D. Zühlke, "Human-MachineInteraction in the Industry 4.0 Era," in 2014 12th IEEE International Conference on Industrial Informatics (INDIN), 2014, pp. 289-294.

[5] S. J. Henderson and S. Feiner, "Evaluating the Benefits of Augmented Reality for Task Localization in Maintenance of an Armored Personnel Carrier Turret," in Proc 8th Int Symp on Mixed and Augmented Reality, 2009, pp. 135-144.

[6] A. L. Janin et al., "Calibration of Head-Mounted Displays for Augmented Reality Applications," in Int Symp Virtual Reality, 1993, pp. 246-255.

[7] S. Feiner et al., "Knowledge-based Augmented Reality," Communications of the ACM, vol. 36, no. 7, pp. 53-62, 1993.

[8] S. Wiedenmaier et al., "Augmented Reality for Assembly Processes. Design and Experimental Evaluation," $H C I$, vol. 16, no. 3, pp. 497514, 2003.

[9] M. Reichert and B. Weber, Enabling Flexibility in Process-Aware Information Systems: Challenges, Methods, Technologies. Springer, 2012.

[10] B. Weber, M. Reichert, W. Wild, and S. Rinderle-Ma, "Providing integrated life cycle support in process-aware information systems," International Journal of Cooperative Information Systems, vol. 18, no. 1, pp. 115-165, March 2009.

[11] M. Reichert and P. Dadam, "Enabling adaptive process-aware information systems with adept2," in Handbook of Research on Business Process Modeling, J. Cardoso and W. van der Aalst, Eds. Hershey, New York: Information Science Reference, March 2009, pp. 173-203.
[12] S. Steinau, K. Andrews, and M. Reichert, "The relational process structure," in 30th Int'l Conference on Advanced Information Systems Engineering (CAiSE 2018), ser. LNCS. Springer, 2018, pp. 53-67.

[13] K. Peffers, T. Tuunanen, M. A. Rothenberger, and S. Chatterjee, "A design science research methodology for information systems research," Journal of management information systems, vol. 24, no. 3, pp. 45-77, 2007.

[14] W. Friedrich, D. Jahn, and L. Schmidt, "ARVIKA-Augmented Reality for Development, Production and Service," in International Symposium on Mixed and Augmented Reality (ISMAR), 2002, pp. 3-4.

[15] N. Mundbrod et al., "Context-Aware Process Injection: Enhancing Process Flexibility by Late Extension of Process Instances," in Proc CoopIS 2015, ser. LNCS, no. 9415. Springer, 2015, pp. 127-145.

[16] F. Gottschalk, W. M. P. Van der Aalst, M. H. Jansen-Vullers, and M. La Rosa, "Configurable workflow models," International Journal of Cooperative Information Systems, vol. 17, no. 02, pp. 177-221, 2008.

[17] A. Hallerbach, T. Bauer, and M. Reichert, "Context-based configuration of process variants," in 3rd International Workshop on Technologies for Context-Aware Business Process Management (TCoB 2008), June 2008, pp. 31-40.

[18] A. Murguzur, X. De Carlos, S. Trujillo, and G. Sagardui, "ContextAware Staged Configuration of Process Variants@Runtime," in Advanced Information Systems Engineering. Springer, 2014, pp. 241-255.

[19] R. Aggarwal, K. Verma, J. Miller, and W. Milnor, "Constraint driven web service composition in meteor-s," in IEEE International Conference on Services Computing (SCC 2004), 2004, pp. 23-30.

[20] O. Saidani and S. Nurcan, "Towards context aware business process modelling," in Workshop on Business Process Modelling, Development, and Support, 2007, p. 1.

[21] M. Rosemann, J. Recker, and C. Flender, "Contextualisation of business processes," International Journal of Business Process Integration and Management, vol. 3, no. 1, pp. 47-60, 2008.

[22] C. Bettini, O. Brdiczka, K. Henricksen, J. Indulska, D. Nicklas, A. Ranganathan, and D. Riboni, "A survey of context modelling and reasoning techniques," Pervasive and Mobile Computing, vol. 6, no. 2, pp. 161 $180,2010$.

[23] M. G. Cimino, B. Lazzerini, F. Marcelloni, and A. Ciaramella, "An adaptive rule-based approach for managing situation-awareness," Expert Systems with Applications, vol. 39, no. 12, pp. 10796 - 10811, 2012.

[24] da Cunha Mattos et al., "A Formal Representation for Context-aware Business Processes," J Comp Ind, vol. 65, no. 8, pp. 1193-1214, 2014.

[25] US Food and Drug Administration et al., "Title 21 Code of Federal Regulations (21 CFR part 11): Electronic Records, Electronic Signatures," URL: www.fda.gov/ora/compliance_ref/part11, 2008.

[26] A. B. Craig, Understanding Augmented Reality: Concepts and Applications. Elsevier, 2013.

[27] M. Billinghurst, A. Clark, G. Lee et al., "A Survey of Augmented Reality," Foundations and Trends of Human-Computer Interaction, vol. 8, no. 2-3, pp. 73-272, 2015.

[28] A. Yilmaz et al., "Object Tracking: A Survey," ACM Computing Surveys (CSUR), vol. 38, no. 4, p. 13, 2006. 